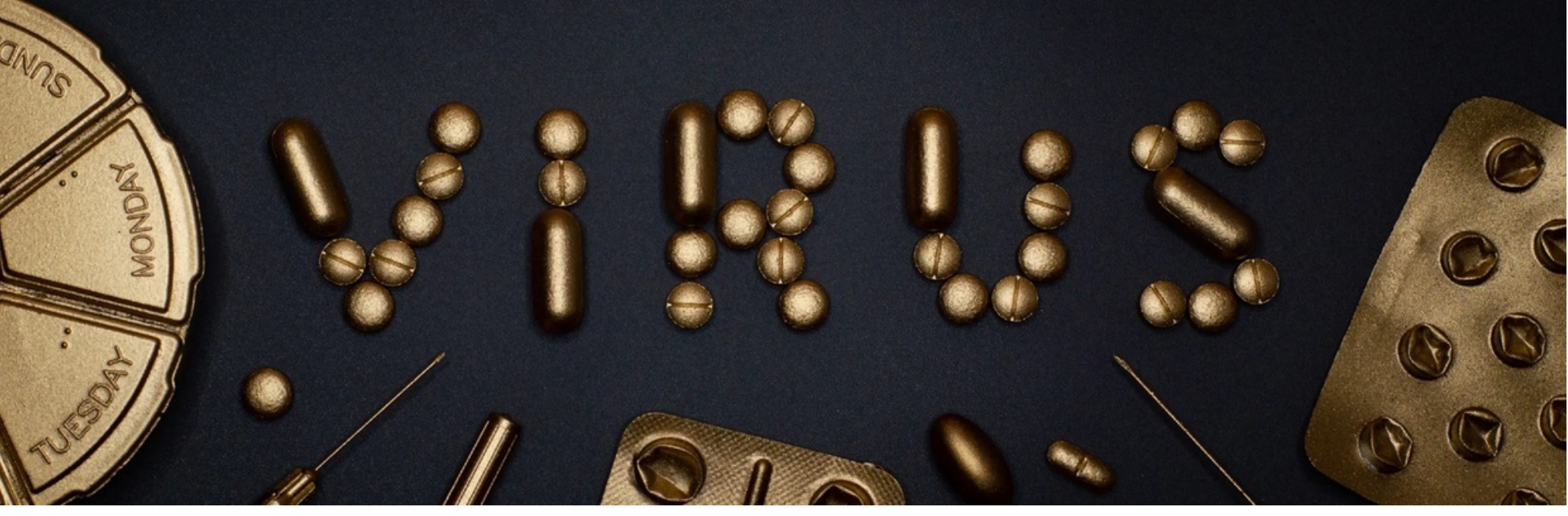

\title{
Enfermedades Crónicas No Transmisibles en Argentina - Chronic Non-Communicable Diseases in Argentina
}

Authors: Gisela Pattarone

Submitted: $\quad$ 24. July 2020

Published: $\quad 27$. July 2020

Volume: 7

Issue: 4

Affiliation: $\quad$ Faculty of Pharmacy and Biochemistry, Faculty of Medicine Universidad de Buenos Aires, Argentina and Faculty of Medicine - Albert Ludwigs University of Freiburg, Germany

Languages: $\quad$ Spanish, Castilian

Keywords: $\quad$ Enfermedades crónicas, prevención, medicina del estilo de vida Categories: Medicine

DOI: $\quad$ 10.17160/josha.7.4.701

Abstract:

A constant change in human health is evident, enhanced by technological advances and the development of scientific research. These constant changes are a setback in terms of the development and persistence of chronic diseases, being these the main causes of mortality and morbidity in Argentina, among which the Chronic Diseases stand out Non-transmissible (NTC) 1. These include cardiovascular disease, diabetes, obesity, metabolic syndrome, kidney disease, certain types of cancer and chronic respiratory disease. It is a situation of enough changes to be able to carry out prevention measures in order to avoid the appearance of these diseases, as well as to improve the treatment indicated by the medical specialist, finally avoiding that they continue to be prolonged in time. This constant change causes a wide field for Evidence-Based Medicine (EBM) 2 that seeks to integrate the different individual clinical experiences with the hest external evidence for decicion making In this field arises the develonment of an effective strateov

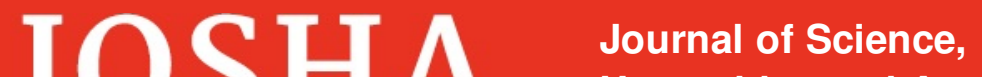 Humanities and Arts}




\title{
Enfermedades Crónicas No Transmisibles en Argentina
}

\author{
Revisión
}

\author{
By Gisela Pattarone. Médica 1 - Maestría en Ciencias 2,3 \\ 1. Facultad de Medicina, Universidad de Buenos Aires (UBA). \\ 2. Facultad de Farmacia y Bioquímica, Universidad de Buenos Aires. \\ 3. Albert-Ludwigs-Universität Freiburg, Alemania.
}

\section{Resumen}

Resulta evidente un constante cambio en materia de salud humana potenciado por los avances tecnológicos y el desarrollo de la investigación científica. Dichos cambios constantes resultan una contracara en términos de desarrollo y persistencia de enfermedades crónicas, siendo estas las principales causas de mortalidad y morbilidad en Argentina, entre las cuales se destacan las Enfermedades Crónicas No Transmisibles (ECNT) ${ }^{1}$. En las mismas se incluyen enfermedades cardiovasculares, diabetes, obesidad, síndrome metabólico, enfermedades renales, ciertos tipos de cáncer y enfermedades respiratorias crónicas. Esta situación de constante cambio se presenta en un terreno firme de conocimiento en lo que respecto a la alteración de la fisiología humana y la fisiopatogenia propia de la enfermedad que prolonga o trae consecuencias en el tiempo. Sin embargo, presenta un segundo escenario de incertidumbre para la toma de decisiones fundamentado por las constantes publicaciones de investigaciones, ensayos clínicos y promociones de nuevos fármacos los cuales se dan en tiempos acelerados, con limitada evidencias $\mathrm{y} / \mathrm{o}$ experiencia de uso. Manteniéndose, de cierta forma, al personal de salud desinformado para poder realizar medidas de prevención con el fin de sortear la aparición de dichas enfermedades, así como para mejorar el tratamiento indicado por el médico especialista, evitando finalmente que se continúen prolongando en el tiempo. Este constante cambio provoca un amplio campo para la Medicina Basada en la Evidencia $(\mathrm{MBE})^{2}$ que busca integrar las diferentes experiencias clínicas individuales con la mejor evidencia externa para la toma de decisiones. En este campo surge el desarrollo de una estrategia efectiva conocida como Medicina del estilo de vida ${ }^{5}$ que busca convertirse en el enfoque principal para el manejo de estas afecciones crónicas y, lo que es más importante, su prevención. Permitiendo 
finalmente la des-prescripción de un tratamiento farmacológico, la reversión de los factores de riesgo biológicos modificables, así como el aprendizaje de hábitos saludables en los factores de riesgo de comportamiento.

Palabras clave: enfermedades crónicas, prevención, medicina del estilo de vida

\section{Introducción}

En los últimos años lo único permanente que se destaca en materia de salud humana es que se encuentra en un constante cambio. Este cambio es fomentado por un mundo con avances tecnológicos en áreas de investigación y producción industrial que implica por sobre todas las cosas tiempos acotados de adaptación y respuesta, en la mayoría no sostenibles en el tiempo. Ya sea en lo referido al desarrollo de una enfermedad que luego se vuelve crónica por la cual comienzan un sinfín de abordajes independientes y hasta ambiguos o presuntamente mágicos que implican resolver patologías de años de permanencia y desarrollo en el cuerpo humano en cuestión de meses. Así como también se presenta el escenarios de contraer una infección o resolverla a través de un nuevo fármaco recientemente comercializado y publicado en todos los medios de comunicación. Esta información rápida y cambiante es tanto una contracara como promotor de la persistencia a lo largo de la historia de la población mundial y por ende, de Argentina de las denominadas Enfermedades Crónicas No Transmisibles (ECNT) ${ }^{1}$. En las mismas se incluyen enfermedades cardiovasculares, diabetes, obesidad, síndrome metabólico, enfermedades renales, ciertos tipos de cáncer y enfermedades respiratorias crónicas. Esta situación genera, en la mayoría de los casos, dos aspectos difíciles de unir. Primero, que el personal de salud dedicado a atender a los pacientes con dichas patologías tengan bases fisiopatológicas certeras. Es decir, transitan un terreno firme en lo que respecta a los hechos de cómo se altera la fisiología humana para dar lugar a la patología y como la fisiopatogenia propia de la enfermedad prolonga o trae consecuencias en el tiempo. Segundo, los constantes cambios, publicaciones de ensayos clínicos y promociones de nuevos fármacos generan situaciones de incertidumbre para la toma de decisiones en base a criterios que no siempre se encuentran perfectamente fundados ya que en tiempos son muy agotadas sus evidencias y/o experiencia. Esto provoca que en materia de salud a lo largo de los últimos años recobre cada vez mayor importancia lo que se conoce como Medicina Basada en la Evidencia (MBE) ${ }^{2}$ debido al constante cambio en materia de investigación un libro publicado en el corriente año probablemente en menos de dos meses se encuentre desactualizado respecto a la información publicada. MBE realiza un intento de integrar las diferentes experiencias clínicas individuales con la mejor evidencia externa para tomar decisiones sobre la atención de los pacientes. 
Se ha proyectado que para el 2025, las ECNT explicarán el 75\% de todas las muertes en el mundo, y queel $71 \%$ de las muertes por enfermedad coronaria, $75 \%$ por enfermedad cerebrovascular y $70 \%$ de las muertes por diabetes se producirán en el mundo en desarrollo ${ }^{3}$.

\section{Argentina y las ECNT}

En nuestro país las Enfermedades Crónicas No Transmisibles son la principal causa de muerte y discapacidad. Se refiere a las enfermedades cardíacas, enfermedad cerebrovascular, diabetes en conjunto con obesidad y síndrome metabólico, enfermedades respiratorias crónicas, enfermedades renales cronicas y entre los tipos de cáncer se encuentran el de pulmón, el colorrectal, el de mama, el de próstata, páncreas, estómago y el de cuello de útero.

Acorde a los datos epidemiológicos publicados por el Ministerio de Salud en la Resolución 1083/2009, sólo dos grupos de causas: cardiovasculares y cáncer, son responsables de la mitad de todas las muertes en Argentina. ${ }^{1}$

Es importante recalcar que no solo en términos de mortalidad sus números son elevados sino que también en morbilidad. En muchos casos donde las enfermedades continúan prolongandose en el tiempo implica años vividos con discapacidad. Lo cual lleva a consecuencias no solo en la salud sino también en la economía propia producto de un mayor gasto, ya sea privado y/o público en instituciones y profesionales de salud. Lo cual lleva implícito una caída en el consumo y el ahorro. Situación que se da frente a posibles restricciones de la oferta laboral y menor productividad debido a limitaciones físicas (Anderson et al. 2009) ${ }^{4}$

\section{Características que tienen en común las ECNT}

El aspecto particular que presentan la mayor parte de este tipo de patologías es que comúnmente se las asocia a la presencia de ciertos factores de riesgo, característicamente, evitables. Estos factores de riesgo compartidos suelen ser las bases fisiopatológicas que explican el desarrollo y la prolongación en el tiempo de las enfermedades. Estos factores se pueden agrupar en:

- Biológicos: sobrepeso, hipertensión arterial, colesterol y lípidos elevados en sangre, diabetes o manejo desregulado de la glucemia en sangre.

- De comportamiento: alimentación no equilibrada, inactividad física, abuso de sustancias como el consumo excesivo de alcohol y tabaco, malos hábitos de sueño. 
- Sociales: falta de desarrollo de estrategias para controlar el estrés, y la ausencia de formar y mantener relaciones interpersonales de calidad para la resiliencia emocional.

En Argentina, el análisis realizado por el Ministerio de Salud teniendo en cuenta Indicadores básicos del 2005 destaca la presencia como agentes causantes 0 determinantes de cronicidad que tienen los factores de riesgo biológicos y de comportamiento sobre las diferentes patologías crónicas.

Referente al sobrepeso y la obesidad, relacionados con la alimentación no saludable y el sedentarismo, menciona la alta prevalencia en nuestro país. Siendo el sobrepeso la base fisiopatológica del $58 \%$ del desarrollo posterior de diabetes, así como el $21 \%$ de las enfermedades coronarias y entre un 8 a $42 \%$ presente como comorbilidad en diversos cánceres como endometrio, mama, próstata y colon. Además, la diabetes es atribuida como responsable del $15 \%$ de los eventos coronarios y el $13 \%$ de las enfermedades renales que requieren asistencia del tipo de diálisis crónicas. A su vez, menciona que la hipertensión arterial explica la fisiopatología del $62 \%$ de la enfermedad cerebrovascular y el $49 \%$ de la enfermedad coronaria. Lo cual se encuentra en relación con los valores altos de colesterol en sangre, como importante factor de riesgo cardiovascular que sería determinante en el desarrollo del $59 \%$ de las enfermedades coronarias y el $18 \%$ de la enfermedad cerebrovascular. La prevalencia en la población estudiada de los diferentes factores de riesgo biológicos puede verse en el Gráfico 1, adaptado de la resolución correspondiente.

\section{Prevalencia de factores de riesgo biológicos ${ }^{1}$}

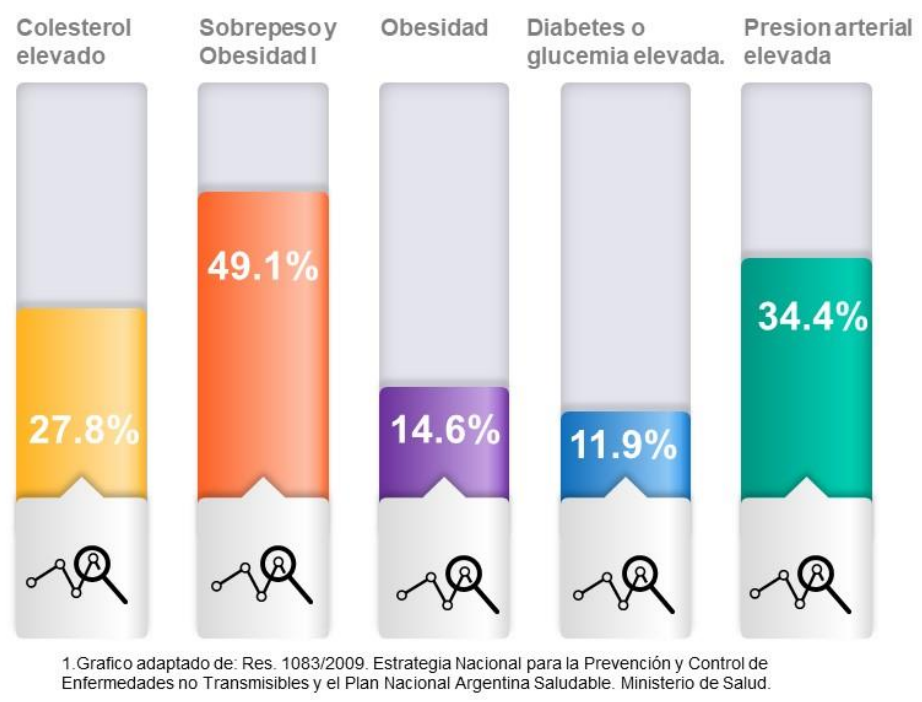


En lo referente a factores de comportamiento o estilo de vida, destacan el tabaquismo como primera causa evitable, asociada con el $12 \%$ de las enfermedades cardiovasculares, $70 \%$ de cánceres de tráquea, bronquios y pulmón y $38 \%$ de la enfermedad respiratoria crónica. En cuanto a la alimentación y abuso de sustancias, analizan la prevalencia que implica una ingesta inadecuada de frutas y verduras se estima que ocasiona el 19\% de los cánceres del tubo digestivo, $31 \%$ de la enfermedad coronaria y $11 \%$ de la enfermedad cerebrovascular junto a la ingesta desmedida y descontrolada de sodio y alcohol como constituyentes principales de las diferentes enfermedades crónicas no transmisibles. Finalmente, la falta de actividad física regular adecuada se estima que causa entre 10 y $16 \%$ de los casos de diferentes cánceres, diabetes, y cerca del $22 \%$ de la enfermedad cardiovascular. La prevalencia en la población estudiada de los diferentes factores de riesgo de estilo de vida puede verse en el Gráfico 2, adaptado de la resolución correspondiente.

\section{Prevalencia de factores de estilo de vida ${ }^{1}$}

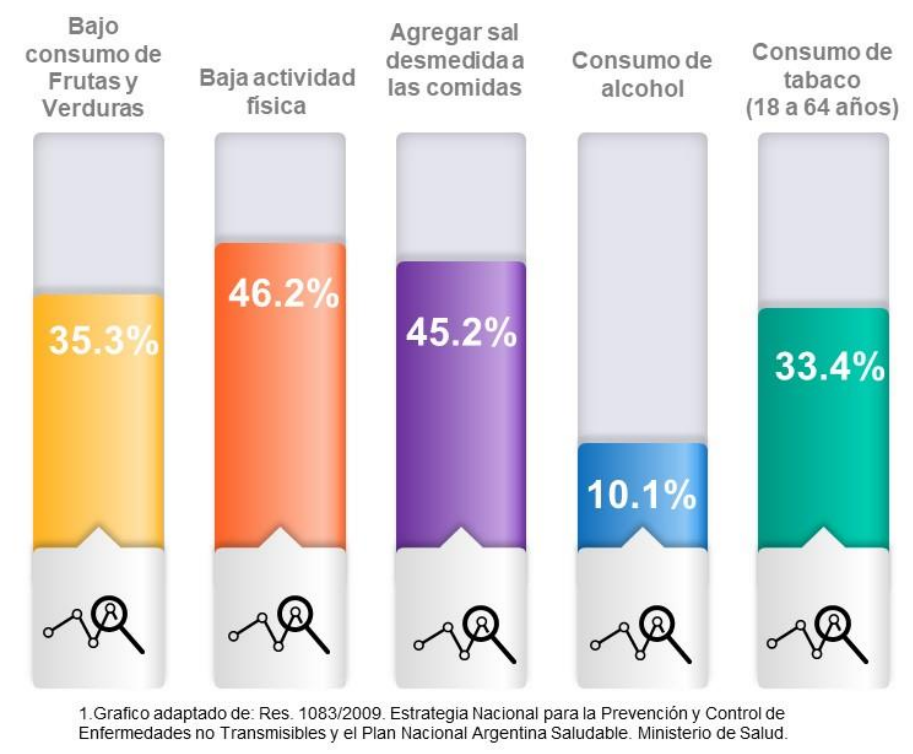

\section{Abordaje para hacer frente a las ECNT}

En la actualidad, en Argentina, se encuentra vigente la denominada Estrategia Nacional para la Prevención y Control de las Enfermedades no transmisibles ${ }^{1}$. Dicha estrategia se encuentra orientada a los siguientes objetivos generales tales como reducir la prevalencia de factores de riesgo de las enfermedades no transmisibles en la población general y reducir su mortalidad asociada. Para alcanzar dichos objetivos generales se centra en tres grandes ejes de acción relacionados con la modificación de los factores determinantes. El primero enfocado a la promoción de hábitos 
saludables como ser acciones de educación, comunicación y concientización de la población, regular y disminuir el tabaquismo, mejorar los hábitos alimenticios. El segundo en relación con los productos de consumo se basa en regular la publicidad, patrocinio y promoción de productos, información al consumidor y normas de etiquetado. Tercero y último, promover entornos saludables que sean favorecedores de actividad física incluyendo infraestructura urbana y de transporte, instituciones, empresas y municipios saludables.

Estas medidas abarcativas y generales distan mucho de la posible planificación de una estrategia diaria para la población, y posibles pacientes en el consultorio médico. Resultando difícil implementarlas como plan de acción individual. Si bien resulta claro que se debe actuar en medidas de prevención a través de un enfoque integrado multidisciplinario deja entre líneas una cierta escala de grises para la toma de decisiones. Perpetuando, en algún punto, las estrategias terapéuticas de indicaciones farmacológicas como primera instancia, sin la llegada de una segunda instancia que determine la posibilidad del retiro de dicho fármaco. Esto mantiene, de cierta forma al personal de salud marginalizado de poder realizar medidas de prevención con el fin de sortear la aparición de dichas enfermedades, así como para acompañar y/o mejorar el tratamiento indicado por el médico especialista, evitando finalmente que se continúen prolongando en el tiempo. Es decir, evita el desarrollo de una estrategia efectiva que permita finalmente la des-prescripción de un tratamiento farmacológico, la reversión de los factores de riesgo biológicos, así como el aprendizaje de nuevos hábitos en los factores de riesgo de comportamiento por parte del paciente y la población general. Es en esta área gris de tratamiento que recobró importancia la mencionada medicina basada en evidencia con una vasta recopilación de publicaciones e investigaciones científicas que al día de hoy se reúnen bajo un enfoque conocido como Medicina del estilo de vida ${ }^{5}$.

\section{Medicina del estilo de vida y Medicina Basada en la Evidencia}

Ambos abordajes resultan fundamentalmente de numerosas publicaciones científicas que abarcan diferentes tipos de estudios epidemiológicos, ecológicos e intervencionistas de las patologías crónicas mencionadas como principal causa de muerte y morbilidad a nivel mundial.

Son numerosos los autores que destacan como campo de acción abordar a los pacientes en la consulta con recomendaciones tales como una alimentación orientada al consumo basado en plantas y alimentos integrales. De esta forma, maximizan el consumo de alimentos ricos en nutrientes y minimizan los productos de origen animal, incluidos los lácteos y los alimentos procesados con azúcar, sal y aceite añadidos. Postulan esta alimentación como sinónimo de nutrición antiinflamatoria. Además, una dieta de alimentos integrales de origen vegetal promueve el aumento del consumo de 
verduras de hoja verde, verduras, frutas, legumbres y granos integrales como alimentos básicos ya que se ha demostrado que los beneficios de una dieta basada en plantas y alimentos integrales influyen sustancialmente en el desarrollo de enfermedades cardiovasculares, así como en muchas enfermedades malignas comunes y actúan sobre los factores de riesgo modificables para enfermedades cardiovasculares y numerosos cánceres (Bodai BI, 2018) ${ }^{5}$.

Además, con el mismo enfoque se han desarrollado metanálisis y revisiones de las evidencias científicas para profundizar el efecto combinado de los diferentes aspectos de la Medicina del estilo de vida sobre la mortalidad de diferentes patologías. Fueron seleccionados estudios prospectivos donde se informaban los efectos combinados del análisis de al menos tres de los siguientes factores: obesidad, consumo de alcohol, tabaquismo, dieta y actividad física. Para analizar el efecto que tienen sobre la mortalidad en conjunto comparándolo con los factores de estilo de vida saludable. El metanálisis comprendió 531804 personas con un seguimiento promedio de 13,24 años y evidenció que para todas las causas de mortalidad los riesgos relativos disminuyeron en proporción al aumento de incorporar factores de estilo de vida saludable. Sentenciando que una combinación de al menos cuatro factores de vida saludable se asocia con una reducción del riesgo de mortalidad por todas las causas en un $66 \%$ (Loef M, 2012). ${ }^{6}$

\section{ECNT y Medicina del estilo de vida}

Se trata de un abordaje de mantener salud y bienestar a través de un estilo de vida saludable y por otro lado prevenir, tratar y revertir las Enfermedades Crónicas No Transmisibles (ECNT). e implica los siguientes puntos:

- Comer de forma saludable

- Aumentar la actividad física

- Mejorar los hábitos de sueño

- Suspender y/o disminuir el abuso de sustancias nocivas

- Desarrollar estrategias para controlar el estrés

- Formar y mantener relaciones interpersonales

Acorde al American College of Lifestyle Medicine ${ }^{7}$ el primer aspecto a abordar en el consultorio médico luego de realizar un examen físico completo y una historia clínica detallada sobre antecedentes médicos de importancia tanto físicos, psicológicos y de laboratorio, es la nutrición actual. Esto implica poder realizar una correcta anamnesis acerca de las preferencias y hábitos alimenticios que llevan adelante los pacientes para poder comenzar con la implementación en este aspecto de elegir alimentos integrales en base a plantas, que tengan alto contenido de fibra, que sean nutritivos, que promuevan el bienestar. Considerando el consumo de verduras, frutas, 
legumbres, granos integrales, frutos secos y semillas, y en caso de ser necesario, eventual consulta con un profesional acorde para un mejor desarrollo del objetivo nutricional.

Sin dejar de lado, realizar una anamnesis correspondiente a los niveles de actividad física diaria que tengan los pacientes y la población general. Teniendo en consideración que el mantenerse activo es lo que se busca fundamentar y esto implica tanto realizar actividad física regular y constante, como así también actividades que puede la población realizar diariamente donde se incluyen tareas como caminar, jardinería, flexiones y estocadas por manipular objetos en el trabajo, como una parte esencial en la ecuación para una salud óptima.

La historia clínica, además, cuenta con un apartado fundamental para los hábitos del sueño. Se encuentra ampliamente documentado que la falta de, o mala calidad de sueño puede llevar a: una disminución en la capacidad de recuperarse de una enfermedad, presentar un sistema inmune exacerbado y mayor predisposición a síntomas como cefalea o fatoga y enfermedades crónicas. La medicina de estilo de vida busca en este sentido identificar comportamientos alimentarios, ambientales y de afrontamiento para mejorar la salud del sueño.

Respecto a los hábitos en relación a consumo de ciertas sustancias es importante tanto informar al paciente como acompañarlo en este aspecto. Se encuentran bien documentados los peligros de cualquier uso adictivo de sustancias que pueden aumentar el riesgo de muchos cánceres y enfermedades del corazón. Se busca entonces desarrollar comportamientos positivos que mejoran la salud e incluyen la cesación del consumo de tabaco y la limitación o ausencia de la ingestión de alcohol.

A diferencia de otros programas y estrategias nacionales, este enfoque implica desarrollar estrategias para aliviar el estrés. Ya que el mismo en sus dos extremos puede guiar al mejoramiento de la salud y productividad, o también a tener ansiedad, depresión, obesidad, disfunción inmune y resultados precarios de salud. Resulta fundamental acompañar y ayudar a los pacientes mediante mecanismos de afrontamiento y técnicas de reducción de estrés, tales como mindfulness que los lleva a reconocer las respuestas negativas del estrés y mejorar su salud y bienestar.

Por último y no por eso menos importante, este enfoque abarca las relaciones interpersonales de la población en general. Resulta clara la importancia de que los pacientes se sientan conectados socialmente. Esto es esencial para la resiliencia emocional y salud en general. Está demostrado por nuestro contexto actual de pandemia que el aislamiento y la soledad se asocian con una mayor mortalidad y morbilidad, especialmente entre las personas que ya han sido diagnosticadas con afecciones relacionadas al estilo de vida. 


\section{Conclusiones}

Es evidente que resulta necesario un cambio de abordaje de la salud en la situación actual de constante cambio que vive un paciente y la población en general, así como la gran incidencia, prevalencia y permanencia en el tiempo de las Enfermedades Crónicas No Transmisibles. Las mismas condicionan en primera instancia a situaciones incapacitantes como días de ausencia laboral, universitario y/o reuniones sociales. Ya sea por la enfermedad misma o porque trae asociado un proceso inflamatorio crónico que provoca síntomas inespecíficos que incluye la debilidad, el malestar, la dificultad de concentración, la depresión y el letargo las cuales provocan emociones negativas habituales consecuentes. Además, este abordaje debe contemplar tanto los estados de enfermedad como así también el mantenimiento del estado de salud, es decir acompañar como profesionales el acto de prevenir la aparición de enfermedades crónicas, ya sea en corto, mediano y/o largo plazo. A toda esta situación se le agrega el impacto económico de un posible menor ingreso como así también aumento de gastos familiares por atención sanitaria. Finalmente la falta de cambio de abordaje de la salud concluye en lo que se observa actualmente que implica consecuencias tales como envejecimiento prematuro, años vividos posiblemente con incapacidad alguna y una mayor probabilidad de mortalidad.

Acorde a las investigaciones, la Medicina del estilo de vida en el consultorio actual resulta una estrategia efectiva que permita acompañar un estado de salud para realizar prevención, tratar los estados de ausencia de salud donde se manifiestan enfermedades crónicas y la reversión en términos de finalmente la des-prescripción de un tratamiento farmacológico, mejorando los factores de riesgo biológicos modificables, así como el aprendizaje de hábitos saludables en los factores de riesgo de comportamiento.

Esta propuesta permite al cuerpo humano protegerse y sanarse a sí mismo a través de elecciones de estilo de vida saludables. Busca educar, guiar y apoyar como profesionales de la salud cambios de comportamiento positivos, no solo en los pacientes sino también en la población en general. Centra su atención en la nutrición óptima basada en la evidencia, manejo del estrés, sueño y actividad física. Alienta la participación activa del paciente acompañado por la contención por parte de un profesional médico de cabecera que conozca en detalle la historia clínica, que incorpore y tenga en consideración dichos detalles como un todo y no como eventos aislados. Además, trata las causas subyacentes de las enfermedades relacionadas al estilo de vida con la principal premisa que utiliza medicamentos como complemento para los cambios de estilo de vida terapéuticos. Considerando en todo momento el entorno del hogar y la comunidad del paciente como importantes. 


\section{Comentarios del autor}

Esta publicación resulta de una motivación propia por la cual creí que debía tomar ciertas medidas de acción que en primera instancia llevé a mi práctica profesional diaria. Intentando fomentar con la misma los valores que adquirí como médica y basándome en el juramento hipocrático que juré defender donde señala "Primero no hacer daño", creí oportuno recopilar esta información apoyándome en mis antecedentes en docencia e investigación con el fin de llegar a la comunidad general y a mis pacientes. Así como también a mis colegas, a quien con el respeto que se merecen los invito a inspirarse, leer publicaciones y realizar investigaciones de Medicina del estilo de vida e incorporar tales prácticas en el cuidado diario de sus pacientes. Creo que el momento de hacer este cambio es ahora.

\section{Referencias}

1.Res. 1083/2009. Estrategia Nacional para la Prevención y Control de Enfermedades no Transmisibles y el Plan Nacional Argentina Saludable. Ministerio de Salud.

2. David L Sackett. Evidence based medicine: what it is and what it isn't. BMJ 1996;312:71.

3. WHO/NMH/NVI/15.1. Informe sobre la situación mundial de las enfermedades no transmisibles 2014.Organización Mundial de la Salud.

4. Anderson, G. F., P. S. Hussey, B. K. Frogner, and H. R. Waters (2005). "Health Spending In The United States And The Rest Of The Industrialized World." Health Affairs, Vol 24 (4): 903-14.

5. Bodai BI, Nakata TE, Wong WT, et al. Lifestyle medicine: A brief review of its dramatic impact on health and survival. Perm J 2018;22:17-025.

6. Loef $\mathrm{M}$, Walach $\mathrm{H}$. The combined effects of healthy lifestyle behaviors on all cause mortality: a systematic review and meta-analysis. Prev Med. 2012;55(3):163-170. doi:10.1016/j.ypmed.2012.06.017.

7. Colegio Estadounidense de Medicina del Estilo de Vida (ACLM por sus siglas en inglés). lifestylemedicine.org 


\section{About the author:}

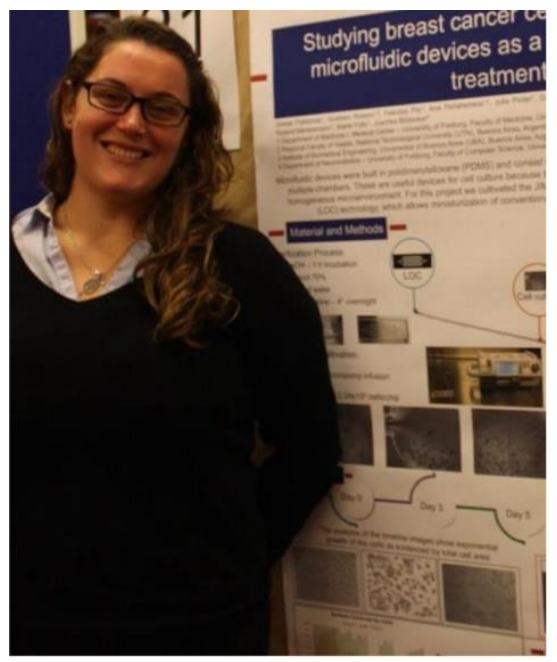

My name is Gisela Pattarone, I am 32 years old. My professional career started in my second year of Medicine. While working academically as a professor in the $3^{\text {rd }}$ department of Anatomy at the University of Buenos Aires, I have improved my skills and acquired deep knowledge. I also developed two of the most important pillars of Medicine in my opinion, the first been Teaching and the Second Research. In fact, I continue to do so today after ten years. After graduating from the School of Medicine in Buenos Aires, Argentina in 2015, I studied english and medicine abroad in Boston, USA, in 2017. One year later, I started my Master in Biomedical Sciences at the University of Buenos Aires, Argentina and the University Albert Ludwig, Freiburg, Germany. Finally obtain my Binational: Argentina and Germany Master Degree in Science in February 2020. Currently, based in Buenos Aires, I am working and studying in Computational Neuroscience of Alzheimer's disease with a highly enthusiastic and challenging PhD program and developing the third important pillars of Medicine: clinical attention. 